ANALYSIS \& PDE Volume 11 No.1 2018

JONATHAN M. FRASER AND TUOMAS SAHLSTEN
ON THE FOURIER ANALYTIC STRUCTURE OF THE BROWNIAN GRAPH 


\title{
ON THE FOURIER ANALYTIC STRUCTURE OF THE BROWNIAN GRAPH
}

\author{
JONATHAN M. FRASER AND TUOMAS SAHLSTEN
}

\begin{abstract}
In a previous article (Int. Math. Res. Not. 2014:10 (2014), 2730-2745) T. Orponen and the authors proved that the Fourier dimension of the graph of any real-valued function on $\mathbb{R}$ is bounded above by 1 . This partially answered a question of Kahane (1993) by showing that the graph of the Wiener process $W_{t}$ (Brownian motion) is almost surely not a Salem set. In this article we complement this result by showing that the Fourier dimension of the graph of $W_{t}$ is almost surely 1. In the proof we introduce a method based on Itô calculus to estimate Fourier transforms by reformulating the question in the language of Itô drift-diffusion processes and combine it with the classical work of Kahane on Brownian images.
\end{abstract}

\section{Introduction and results}

1A. Geometric properties of Brownian motion. Gaussian processes are standard models in modern probability theory and perhaps the most well-studied example is the Wiener process (or standard Brownian motion) $W=W_{t}: \mathbb{R}^{\geqslant 0} \rightarrow \mathbb{R}$ characterised by the properties $W_{0}=0$, the map $t \mapsto W_{t}$ is almost surely continuous, and $W_{t}$ has independent increments such that $W_{t}-W_{s}$ for $t>s$ is normally distributed:

$$
W_{t}-W_{s} \sim N(0, t-s) .
$$

The Wiener process has far-reaching importance throughout mathematics and it is a topic of particular interest to understand its geometric structure. This can be achieved by studying several random fractals associated to the process such as images $W(K):=\left\{W_{t}: t \in K\right\}$ of compact sets $K \subset[0, \infty)$, level sets $L_{c}(W):=\left\{t \in \mathbb{R}: W_{t}=c\right\}$ for $c \in \mathbb{R}$, graphs $G(W):=\left\{\left(t, W_{t}\right): t \in \mathbb{R}\right\}$ (see Figure 1) and other more delicate constructions such as $\mathrm{SLE}_{\kappa}$-curves.

The basic properties of Brownian motion mean that these random fractals enjoy a certain "statistical self-similarity", which facilitates computation of their Hausdorff dimensions $\operatorname{dim}_{\mathrm{H}}$. Classical results include McKean's proof [1955] that $\operatorname{dim}_{\mathrm{H}} W(K)=\min \left\{1,2 \operatorname{dim}_{\mathrm{H}} K\right\}$ almost surely for each compact $K \subset[0, \infty)$. Moreover, for the level sets, $\operatorname{dim}_{\mathrm{H}} L_{c}(W)=\frac{1}{2}$ almost surely for $c=0$ by [Taylor 1955] and for all $c \in \mathbb{R}$ by [Perkins 1981] conditioned on $L_{c}(W)$ being nonempty. For the Brownian graph $G(W)$, Taylor [1953] proved that $\operatorname{dim}_{\mathrm{H}} G(W)=\frac{3}{2}$ almost surely and Beffara [2008] computed the Hausdorff dimensions of $\mathrm{SLE}_{\kappa}$-curves. Moreover, Hausdorff dimensions for similar sets given by many other Gaussian processes, such as fractional Brownian motion, have been also considered; see, for example,

Fraser acknowledges financial support from the Leverhulme Trust (RF-2016-500) and Sahlsten acknowledges the support from the European Union (ERC grant no. 306494 and Marie Skłodowska-Curie Individual Fellowship grant no. 655310).

MSC2010: primary 42B10, 60H30; secondary 11K16, 60J65, 28A80.

Keywords: Brownian motion, Wiener process, Itô calculus, Itô drift-diffusion process, Fourier transform, Fourier dimension,

Salem set, graph. 


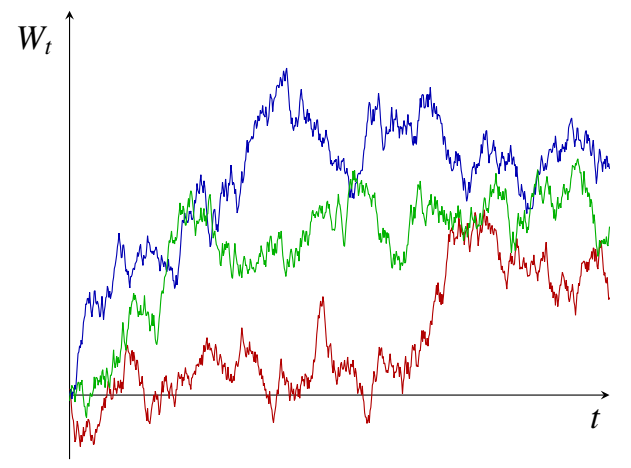

Figure 1. Three realisations of the graph $G(W)$ for the Brownian motion $W_{t}$.

Adler's classical results [1977] for fractional Brownian graphs and the recent work [Peres and Sousi 2016] concerning variable drift.

1B. Fourier analytic properties of Brownian motion. The Hausdorff dimension is the most commonly used tool for measuring the size of a set $A$ but there is also another fundamental notion based on Fourier analysis which reveals more arithmetic and geometric features of $A$, including curvature, which are not seen by the Hausdorff dimension. This is based on studying the Fourier coefficients of a probability measure $\mu$ on $A \subset \mathbb{R}^{d}$, which are defined by

$$
\hat{\mu}(\xi):=\int e^{-2 \pi i \xi \cdot x} d \mu(x), \quad \xi \in \mathbb{R}^{d} .
$$

Now the size of $A$ can be linked to the existence of probability measures $\mu$ on $A$ with decay of Fourier coefficients $\hat{\mu}(\xi)$ when $|\xi| \rightarrow \infty$. The following connection between Hausdorff dimension and decay of Fourier coefficients is well known and goes back to Salem and Kaufman, but we refer the reader to [Mattila 2015] for the details. If $\operatorname{dim}_{\mathrm{H}} A>s$, then $A$ supports a probability measure $\mu$ with $|\hat{\mu}(\xi)|=O\left(|\xi|^{-s / 2}\right)$ "on average", that is, $\int_{\mathbb{R}^{d}}|\hat{\mu}(\xi)|^{2}|\xi|^{s-d} d \xi<\infty$, and vice versa the Hausdorff dimension can be bounded from below if such a measure $\mu$ can be found. It is possible, however, that $\operatorname{dim}_{\mathrm{H}} A=s>0$ but no measure $\mu$ on $A$ has Fourier decay at infinity; this happens for example when $A$ is the middle-third Cantor set in $\mathbb{R}$. Therefore, one defines the notion of Fourier dimension $\operatorname{dim}_{\mathrm{F}} A$ of a set $A \subset \mathbb{R}^{d}$ as the supremum of $s \in[0, d]$ for which there exists a probability measure $\mu$ supported on $A$ such that

$$
|\hat{\mu}(\xi)|=O\left(|\xi|^{-s / 2}\right) \quad \text { as }|\xi| \rightarrow \infty
$$

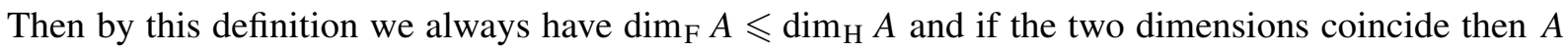
is called a Salem set or a round set after [Kahane 1993]. In general Fourier dimension and Hausdorff dimension have no relationship other than this; in fact, Körner [2011] established that for any $0 \leqslant s<t \leqslant 1$ it is possible to construct examples $A \subset \mathbb{R}$ with $\operatorname{dim}_{\mathrm{F}} A=s$ and $\operatorname{dim}_{\mathrm{H}} A=t$. Further properties of Fourier dimension were recently developed by Ekström, Persson and Schmeling [Ekström et al. 2015]. For a more in depth account of Fourier dimension, the reader is referred to [Mattila 1995; 2015]. 
Finding measures $\mu$ on $A$ with polynomially decaying Fourier transform (i.e., (1-1) for some $s>0$ ) has deep links to absolute continuity, arithmetic and geometric structure, and curvature. If $A$ supports a measure $\mu$ such that (1-1) holds with $s>1$, then Parseval's identity yields that $\mu$ is absolutely continuous to Lebesgue measure and $A$ must contain an interval. An application of Weyl's criterion known as the Davenport-Erdős-LeVeque criterion [Davenport et al. 1963] yields that in $\mathbb{R}$ polynomial decay of $\hat{\mu}$ guarantees that $\mu$ almost every number is normal in every base and an interesting result of Łaba and Pramanik [2009] shows that if the $s$ in (1-1) is sufficiently close to 1 for a Frostman measure $\mu$ on $A \subset \mathbb{R}$ and there is a suitable control over the constants, see the recent work [Shmerkin 2017], then $A$ contains nontrivial 3-term arithmetic progressions. Moreover, an analogous result also holds for higher dimensions with arithmetic patches [Chan et al. 2016].

On the curvature side, if $A$ is a line-segment in $\mathbb{R}^{2}$, then $A$ cannot contain any measure with Fourier decay at infinity so $A$ cannot be a Salem set. However, if $A$ is an arc of a circle or more generally a 1-dimensional smooth manifold with nonvanishing curvature then the 1-dimensional Hausdorff measure $\mu$ on $A$ satisfies (1-1) with $s=1$; see [Mattila 2015]. In particular, $A$ is a Salem set. In these examples of $A$ one can observe that the important arithmetic or curvature features present are not seen from the Hausdorff dimension.

Constructing explicit Salem sets (which are not manifolds), or just sets $A$ supporting a measure $\mu$ satisfying (1-1) for some $s>0$, can be achieved through, for example, Diophantine approximation by [Kaufman 1980; 1981; Bluhm 1998; Queffélec and Ramaré 2003] or via thermodynamical tools by [Jordan and Sahlsten 2016]. However, for random sets it has been observed in many instances that $A$ is either almost surely Salem or at least supports a measure $\mu$ with (1-1) for some $s>0$. This was first done for random Cantor sets by Salem [1951], where Salem sets were also introduced. Later in his classical papers, Kahane [1966a; 1966b] found out that the Wiener process and other Gaussian processes provide natural examples.

Since Kahane and Salem, the study of Fourier analytic properties of natural sets derived from Gaussian processes and more general random fields has been an active topic. For the Brownian images, Kahane [1985b] proved that for any compact $K \subset \mathbb{R}$ the image $W(K)$ is almost surely a Salem set of Hausdorff dimension $\min \{1,2 \operatorname{dim} K\}$. Kahane also established a similar result for fractional Brownian motion. Łaba and Pramanik [2009] then applied these to the additive structure of Brownian images. Later Shieh and Xiao [2006] extended Kahane's work to very general classes of Gaussian random fields. However, understanding the Fourier analytic properties of the level sets and graphs remained an important problem for some time. Kahane [1993] outlined the problem explicitly.

Problem 1.1 (Kahane). Are the graph and level sets of a stochastic process, such as fractional Brownian motion, Salem sets?

This precise formulation of the problem was given by Shieh and Xiao [2006, Question 2.15], but they attribute the problem to Kahane. For the Wiener process Kahane [1985a] had already established that the level sets $L_{c}(W)$ are Salem almost surely for any fixed $c \in \mathbb{R}$ conditioned on $L_{c}(W)$ being nonempty. The fractional Brownian motion case has recently been considered for $c=0$ by Fouché and Mukeru [2013].

Kahane's problem for graphs, even in the case of the standard Brownian motion $W_{t}$, however, remained open for quite a while until, together with T. Orponen, we established that the Brownian graph $G(W)$ is 
almost surely not a Salem set [Fraser et al. 2014]. It turned out that the reason for this is purely geometric: the proof was based on the following application of a Fourier-analytic version of Marstrand's slicing lemma.

Theorem 1.2 [Fraser et al. 2014, Theorem 1.2]. For any function $f:[0,1] \rightarrow \mathbb{R}$ the Fourier dimension of the graph $G(f)$ cannot exceed 1.

Indeed, since $\operatorname{dim}_{H} G(W)=\frac{3}{2}>1$ almost surely [Taylor 1953], this answers Kahane's problem in the negative for the Wiener process. Note that this also gives a negative answer for fractional Brownian motion since the Hausdorff dimension in that case is also strictly larger than 1 almost surely.

The methods in [Fraser et al. 2014] are purely geometric and involve no stochastic properties of Brownian motion. They also do not shed any light on the precise value for the Fourier dimension of $G(W)$. Note that even though $\operatorname{dim}_{\mathrm{H}} G(f) \geqslant 1$ for any continuous $f:[0,1] \rightarrow \mathbb{R}$, the Fourier dimension of a graph may take any value in the interval $[0,1]$; see [loc. cit.]. For example, $\operatorname{dim}_{\mathrm{F}} G(f)=0$ if $f$ is affine and, moreover, $\operatorname{dim}_{\mathrm{F}} G(f)=0$ for the Baire generic $f \in C[0,1]$; see [loc. cit., Theorem 1.3].

The main result of this paper is to complete the work initiated by Kahane's problem in the case of Brownian motion by establishing the precise almost sure value of the Fourier dimension of $G(W)$.

Theorem 1.3. The graph $G(W)$ has Fourier dimension 1 almost surely.

Moreover, the random measure $\mu$ we use to realise the Fourier dimension is Lebesgue measure $d t$ on $[0,1]$ lifted onto the graph $G(W)$ via the mapping $t \mapsto\left(t, W_{t}\right)$. The precise estimate we obtain is that almost surely

$$
|\hat{\mu}(\xi)|=O\left(|\xi|^{-1 / 2} \sqrt{\log |\xi|}\right) \quad \text { as }|\xi| \rightarrow \infty,
$$

which combined with Theorem 1.2 yields Theorem 1.3.

A natural direction in which to continue this line of research would be to study other Gaussian processes with different covariance structure, such as the fractional Brownian motion.

1C. Methods: Itô calculus and reduction to Brownian images. The key method we introduce to estimate the Fourier transform of the graph measure $\mu$ is based on Itô calculus, which has previously been a natural framework in the theory of stochastic differential equations. As far as we know, Itô calculus has not been previously considered in this Fourier analytic context. Here we discuss this method and give a brief summary of the main steps in the proof. When written in polar coordinates, (1-2) asks about the rate of decay for the integral

$$
\hat{\mu}(\xi)=\int_{0}^{1} \exp \left(-2 \pi i u\left(t \cos \theta+W_{t} \sin \theta\right)\right) d t
$$

for $\xi=u(\cos \theta, \sin \theta) \in \mathbb{R}^{2}, u>0, \theta \in[0,2 \pi)$, as $u \rightarrow \infty$. There are two distinct cases we will consider depending on the direction of $\xi$, which we give a heuristic description of here.

If we ignore the random component $W_{t} \sin \theta$, that is, set $\theta=0$ or $\pi$, then standard integration using the chain rule shows that $\hat{\mu}(\xi)$ equals the Fourier transform of Lebesgue measure $d t$ at $u$, which decays to 0 with the polynomial rate $u^{-1}=|\xi|^{-1}$, so we are done for these directions. However, if $\theta$ is not equal to 0 
or $\pi$, we still have a small random (nonsmooth) term $W_{t} \sin \theta$, so a classical change of variable formula or other tools from classical analysis cannot be used.

The key observation is that we can write $\hat{\mu}(\xi)=\int \exp \left(i X_{t}\right) d t$, where the stochastic process $X_{t}$ satisfies the stochastic differential equation

$$
d X_{t}:=b d t+\sigma d W_{t},
$$

identifying it as a so-called Itô drift-diffusion process, where $b:=-2 \pi u \cos \theta$ is the drift coefficient of $X_{t}$ and $\sigma:=-2 \pi u \sin \theta$ is the diffusion coefficient of $X_{t}$. Such processes have many useful analytic tools from Itô calculus (see Section 2) associated to them, in particular Itô's lemma, which works as an analogue for the chain rule. The price we pay is that Itô's lemma introduces some multiplicative error terms involving stochastic integrals, but they can be estimated with other tools from Itô calculus using moment analysis.

The estimates we obtain from Itô calculus allow us to obtain the correct Fourier decay (1-2) for $\mu$ when $\theta$ is close to 0 or $\pi$ with respect to $u^{-1}$ (more precisely, $|\sin \theta|<u^{-1 / 2}$ ), in other words, when $\xi$ is close to pointing in the horizontal directions. Thus another estimate is needed for $\theta$ bounded away from 0 and $\pi$. This is where the classical work [Kahane 1985b] on Brownian images comes into play. If we completely ignore the deterministic component $t \cos \theta$, by setting $\theta=\frac{\pi}{2}$ or $\frac{3 \pi}{2}$, then $\hat{\mu}(\xi)$ is the Fourier transform of the Brownian image measure $v$, that is, the $t \mapsto W_{t}$ push-forward of the Lebesgue measure $d t$ on $[0,1]$ at $u$. Kahane [1985b] in fact already established that the decay of $|\hat{v}(u)|$ is almost surely of the order $u^{-1} \sqrt{\log u}=|\xi|^{-1} \sqrt{\log |\xi|}$ so (1-2) holds for these directions. A modification of Kahane's argument reveals that whenever $\theta \neq 0$ or $\pi$, then almost surely

$$
|\hat{\mu}(\xi)|=O\left(|\sin \theta|^{-1}|\xi|^{-1} \sqrt{\log |\xi|}\right) ;
$$

see the discussion in Section 3C. Now one notices that when $\theta$ approaches 0 or $\pi$, this estimate blows up, and so one cannot obtain a uniform estimate over all directions from this. However, this gives (1-2) if $|\sin \theta| \geqslant u^{-1 / 2}$, so combining with the estimates we obtained through Itô calculus, we are done. See Section 3 for more details on the main steps of the proof.

1D. Other measures on the Brownian graph. Theorem 1.3 and (1-2) give Fourier decay for the pushforward of the Lebesgue measure on $[0,1]$ onto the graph $G(W)$. It would be an interesting problem to see if one can have similar results for other, possibly fractal, measures on $[0,1]$. A possible problem could be:

Problem 1.4. Classify measures $\tau$ on $[0,1]$ such that for some $0<s \leqslant 1$ we have

$$
|\hat{\tau}(\xi)|=O\left(|\xi|^{-s / 2}\right), \quad|\xi| \rightarrow \infty,
$$

and their lift $\mu_{\tau}$ onto the graph of $G(W)$ under $t \mapsto\left(t, W_{t}\right)$ satisfies

$$
\left|\hat{\mu}_{\tau}(\xi)\right|=O\left(|\xi|^{-s^{\prime} / 2}\right), \quad|\xi| \rightarrow \infty
$$

for any $s^{\prime}<s$.

This is motivated by the fact that in [Kahane 1985b] it is possible to transfer information on the Fourier decay (or Frostman properties) of $\tau$ onto the image measure. Thus for directions $\theta$ bounded away from 0 
and $\pi$ we could still bound $\hat{\mu}_{\tau}(\xi)$ using Kahane's work. The main problem in generalising our approach to fractal measures $\tau$ on $[0,1]$ comes from the lack of an appropriate analogue of Itō calculus.

1E. Organisation of the paper. In Section 2 we give the necessary background from Itô calculus. In Section 3 we will give the proof of our main result Theorem 1.3. The key estimates are obtained in Section $3 \mathrm{~B}$ and Section $3 \mathrm{C}$, corresponding to the two cases discussed above.

\section{Itō calculus}

2A. Stochastic integration. In the proof of the main result Theorem 1.3, we end up studying integrals of the form $\int f\left(X_{t}\right) d t$ for some stochastic processes $X_{t}$ and smooth scalar functions $f$. As standard analysis methods cannot be applied to these integrals, we need theory from stochastic analysis. Stochastic analysis provides a pleasant framework to deal with nonsmooth processes, such as the Wiener process $W_{t}$, and still preserves many of the classical features present in the smooth setting. In this section we discuss the specific tools from Itô calculus which we will rely on. The main references for this section are given in the book [Karatzas and Shreve 1991].

Let $\left(\Omega, \mathcal{F},\left(\mathcal{F}_{t}\right)_{t \geqslant 0}, \mathbb{P}\right)$ be a filtered probability space; that is, $\mathcal{F}_{t} \subset \mathcal{F}$ is an increasing filtration in $t$. Let $W=W_{t}$ be the Wiener process adapted to this filtered probability space; that is, $W_{t}$ is $\mathcal{F}_{t}$ measurable and for each $t, s \geqslant 0$ the increment $W_{t+s}-W_{t}$ is independent of $\mathcal{F}_{t}$. We say that an $\mathbb{R}$ - or $\mathbb{C}$-valued stochastic process $Z_{t}$ is adapted if it is $\mathcal{F}_{t}$ measurable for all $t \geqslant 0$. We will say that a real- or complex-valued adapted process $Z_{t}$ is $W_{t}$-integrable if the quadratic variation $\int_{0}^{T}\left|Z_{t}\right|^{2} d t$ is finite for any time $T \geqslant 0$. Given a real-valued adapted $W_{t}$-integrable stochastic process $X_{t}$, we have $\mathbb{P}$ almost surely for any time $T \geqslant 0$ it is possible to construct a stochastic integral

$$
\int_{0}^{T} X_{t} d W_{t}
$$

of $X_{t}$ with respect to $W_{t}$ in the sense of Itô; see [Karatzas and Shreve 1991, Chapter 3.2]. We use the differential notation $d U_{t}=X_{t} d W_{t}$ to mean that $\mathbb{P}$ almost surely $U_{T}-U_{0}$ is the stochastic integral of $X_{t}$ with respect to $W_{t}$ at time $T \geqslant 0$.

We mainly deal with complex-valued stochastic processes, so for the sake of convenience we will also introduce the complex-valued stochastic integral for a $\mathbb{C}$-valued $W_{t}$-integrable adapted process $Z_{t}$, defined coordinatewise using real integrals:

$$
\int_{0}^{T} Z_{t} d W_{t}:=\int_{0}^{T} \operatorname{Re} Z_{t} d W_{t}+i \int_{0}^{T} \operatorname{Im} Z_{t} d W_{t},
$$

where the real integrals are standard $\mathbb{R}$-valued stochastic integrals with respect to the Wiener process $W_{t}$. We write $d Z_{t}:=d X_{t}+i d Y_{t}$ for a complex-valued process $Z_{t}=X_{t}+i Y_{t}$ with $\mathbb{R}$-valued $X_{t}$ and $Y_{t}$.

2B. Itō drift-diffusion processes. The main class of adapted processes to which we apply Itô calculus is given by Wiener processes with drift and diffusion coefficients. These are called Itô drift-diffusions:

Definition 2.1 (Itô drift-diffusion process). A real- or complex-valued adapted stochastic process $X_{t}$ is called an Itô drift-diffusion process if there exists a Lebesgue integrable adapted $b_{t}$ and $W_{t}$-integrable 
adapted $\sigma_{t}$ such that $X_{t}$ satisfies the stochastic differential equation

$$
d X_{t}=b_{t} d t+\sigma_{t} d W_{t} .
$$

For Itô drift-diffusion processes there exists the following important analogue of the change of variable formula, which follows from robustness of Taylor expansions for stochastic differentials:

Lemma 2.2 (Itō's lemma). Let $X_{t}$ be an Itô drift-diffusion process and $f: \mathbb{R} \rightarrow \mathbb{R}$ twice differentiable. Then $f\left(X_{t}\right)$ is an Itô drift-diffusion process such that $\mathbb{P}$ almost surely for any $T \geqslant 0$ we have

$$
f\left(X_{T}\right)-f\left(X_{0}\right)=\int_{0}^{T}\left(b_{t} f^{\prime}\left(X_{t}\right)+\frac{1}{2} \sigma_{t}^{2} f^{\prime \prime}\left(X_{t}\right)\right) d t+\int_{0}^{T} \sigma_{t} f^{\prime}\left(X_{t}\right) d W_{t} .
$$

Itō's lemma was given in this pathwise form in [Karatzas and Shreve 1991, Theorem 3.3]. By using the definition of the complex-valued stochastic integral, we can also obtain a complex-valued Itô's lemma:

Lemma 2.3 (complex Itō's lemma). Let $X_{t}$ be an Itô drift-diffusion process and $f: \mathbb{R} \rightarrow \mathbb{C}$ twice differentiable. Then $f\left(X_{t}\right)$ is an Itô drift-diffusion process such that for $\mathbb{P}$ almost surely for any $T \geqslant 0$ we have

$$
f\left(X_{T}\right)-f\left(X_{0}\right)=\int_{0}^{T}\left(b_{t} f^{\prime}\left(X_{t}\right)+\frac{1}{2} \sigma_{t}^{2} f^{\prime \prime}\left(X_{t}\right)\right) d t+\int_{0}^{T} \sigma_{t} f^{\prime}\left(X_{t}\right) d W_{t} .
$$

Proof. We can write $f=f_{1}+i f_{2}$ for real-valued twice differentiable $f_{1}, f_{2}: \mathbb{R} \rightarrow \mathbb{R}$. Then the derivatives satisfy $f^{\prime}=f_{1}^{\prime}+i f_{2}^{\prime}$ and $f^{\prime \prime}=f_{1}^{\prime \prime}+i f_{2}^{\prime \prime}$. Moreover, by Itô's lemma (Lemma 2.2) we obtain for each $j=1,2$ that

$$
d f_{j}\left(X_{t}\right)=\left(b_{t} f_{j}^{\prime}\left(X_{t}\right)+\frac{1}{2} \sigma_{t}^{2} f_{j}^{\prime}\left(X_{t}\right)\right) d t+\sigma_{t} f_{j}^{\prime}\left(X_{t}\right) d W_{t} .
$$

Then by the convention $d f\left(X_{t}\right)=d f_{1}\left(X_{t}\right)+i d f_{2}\left(X_{t}\right)$ this gives

$$
d f\left(X_{t}\right)=\left(b_{t} f^{\prime}\left(X_{t}\right)+\frac{1}{2} \sigma_{t}^{2} f^{\prime \prime}\left(X_{t}\right)\right) d t+\sigma_{t} f^{\prime}\left(X_{t}\right) d W_{t}
$$

as required.

2C. Moment estimation. Itô's lemma allows us to pass from integrals of the form $\int_{0}^{T} f\left(X_{t}\right) d t$ to $\int_{0}^{T} g\left(X_{t}\right) d W_{t}$ for functions $g$ obtained from derivatives of $f$. In our case we will end up trying to understand the higher moments of the stochastic integrals $\int_{0}^{T} g\left(X_{t}\right) d W_{t}$, which will tell us about the distribution of these integrals. A very standard tool to compute the moments in Itô calculus are the Itô isometry and more general Burkholder-Davis-Gundy inequalities [Burkholder et al. 1972], which allow us to pass from stochastic integrals to their quadratic variations (that just involve Lebesgue integral).

Lemma 2.4 (Burkholder-Davis-Gundy inequality). Let $X_{t}$ be a real-valued $W_{t}$-integrable adapted process. Then for all $1 \leqslant p<\infty$ we have

$$
\mathbb{E}\left[\left(\sup _{0 \leqslant s \leqslant 1}\left|\int_{0}^{s} X_{t} d W_{t}\right|\right)^{2 p}\right] \leqslant 2 \sqrt{10 p} \mathbb{E}\left[\left(\int_{0}^{1} X_{t}^{2} d t\right)^{p}\right] .
$$

This version with the constant $2 \sqrt{10 p}$ was given by Peškir [1996]. 


\section{Proof of the main result}

3A. Preliminaries and overview of the proof. Let us now review how we will prove (1-2) and thus Theorem 1.3. Fix $\xi=u(\cos \theta, \sin \theta) \in \mathbb{R}^{2}$ with modulus $u>0$ and argument $\theta \in[0,2 \pi)$. Notice that by the definition of the graph measure $\mu$, the Fourier transform has the form

$$
\hat{\mu}(\xi)=\int_{0}^{1} \exp \left(i X_{t}\right) d t
$$

where $X_{t}$ is the real-valued stochastic process

$$
X_{t}:=-2 \pi u\left(t \cos \theta+W_{t} \sin \theta\right) .
$$

The first observation is that $X_{t}$ is an adapted $W_{t}$-integrable process and in fact an Itô drift-diffusion process (recall Definition 2.1) satisfying

$$
d X_{t}=b d t+\sigma d W_{t}
$$

for deterministic and time independent coefficients $b=-2 \pi u \cos \theta$ and $\sigma=-2 \pi u \sin \theta$. The proof of bounding $\hat{\mu}(\xi)$ will heavily depend on the value of the angle $\theta$ we have for $\xi$ and in particular how close the determining angle $\theta$ is to $0, \pi$ or $2 \pi$ with respect to $u^{-1 / 2}$. For this purpose, we define the notions of horizontal and vertical angles:

Definition 3.1 (horizontal and vertical angles). Define the threshold angle

$$
\theta_{u}:=\min \left\{u^{-1 / 2}, \frac{\pi}{4}\right\} .
$$

Partition the angles $[0,2 \pi)$ using $\theta_{u}$ into the horizontal angles

$$
H_{u}:=\left[0, \theta_{u}\right] \cup\left[\pi-\theta_{u}, \pi+\theta_{u}\right] \cup\left[2 \pi-\theta_{u}, 2 \pi\right)
$$

and the vertical angles

$$
V_{u}:=[0,2 \pi) \backslash H_{u} .
$$

In other words $H_{u}$ contains the $\theta_{u}$ neighborhoods of 0 and $\pi$ on the circle mod $2 \pi$ and $V_{u}$ the $\frac{\pi}{2}-\theta_{u}$ neighborhoods of $\frac{\pi}{2}$ and $\frac{3 \pi}{2}$ respectively; see Figure 2 .

The proof will split into two cases in Sections 3B and 3C for bounding the Fourier transform $\hat{\mu}(\xi)$ depending on whether $\theta \in H_{u}$ or $\theta \in V_{u}$ :

(1) Section 3B concerns angles $\theta \in H_{u}$, that is, close to horizontal directions 0 or $\pi$, and as mentioned in the Introduction our main hope here is that the smallness (with respect to $u^{-1 / 2}$ ) of the diffusion component $b W_{t}$ will help us in transferring the decay of Lebesgue measure to the decay of $\hat{\mu}$. This is where Itô's lemma (see Lemma 2.3) becomes crucial as it can be applied to the process $f\left(X_{t}\right)$ with the function $f(x)=\exp (i x)$.

(2) Section $3 C$ handles the angles $\theta \in V_{u}$ and here the plan is to use the fact that we are $u^{-1 / 2}$-bounded away from horizontal angles to ignore the drift component $b t$ of the drift-diffusion process $X_{t}$ and apply Kahane's bound for these directions. This turns out to be possible due to a representation of the higher moments Kahane obtained in his result on Brownian images. 


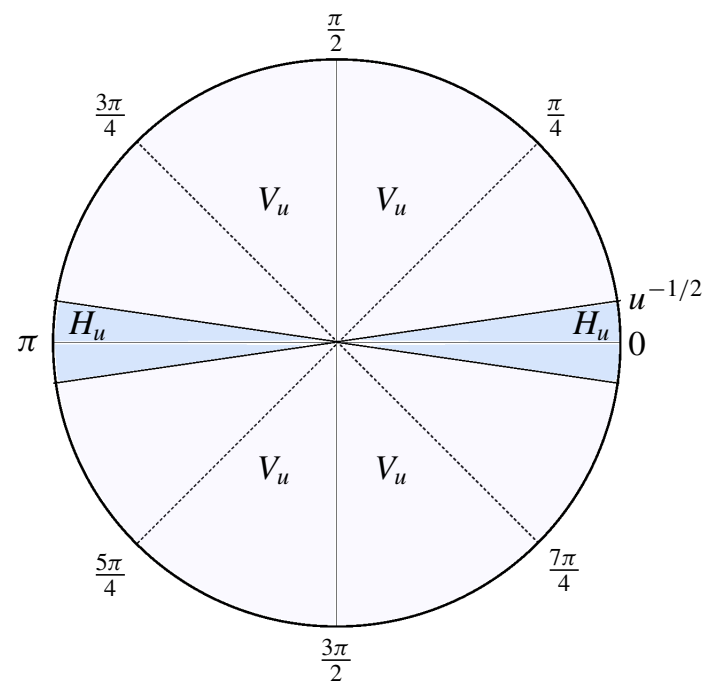

Figure 2. Splitting of $[0,2 \pi)$ to horizontal angles $H_{u}$ and the vertical angles $V_{u}$.

It turns out that in both Sections 3B and 3C we only obtain decay of the Fourier transform $\hat{\mu}(\boldsymbol{k})$ for $\boldsymbol{k}$ in an $\varepsilon$-grid $\varepsilon \mathbb{Z}^{2}$ for all small $\varepsilon>0$. Here the randomness will depend on $\varepsilon>0$ but thanks to an argument also used by Kahane [1985b], one can pass from this information to the full decay almost surely. See Section 3D for the details.

Let us now proceed to bound $|\hat{\mu}(\xi)|$. In both Sections 3B and 3C below we will end up bounding trigonometric functions with respect to $\theta_{u}$ and for this purpose we will need the following standard bounds, which we record here for convenience:

Lemma 3.2 (trigonometric bounds). We have the following bounds:

(1) If $\theta \in H_{u}$, then

$$
|\sin \theta| \leqslant u^{-1 / 2} \text { and } \quad|\cos \theta| \geqslant \frac{1}{\sqrt{2}}
$$

(2) If $\theta \in V_{u}$, then

$$
|\sin \theta| \geqslant \min \left\{\frac{2}{\pi} u^{-1 / 2}, \frac{1}{\sqrt{2}}\right\} .
$$

Proof. For $\alpha \in\left[0, \frac{\pi}{2}\right]$ we have that both cosine and sine are nonnegative. Moreover, here $\frac{2}{\pi} \alpha \leqslant \sin \alpha \leqslant \alpha$. Thus for $\theta \in\left[0, \theta_{u}\right]$ we have

$$
\sin \theta \leqslant \theta \leqslant \theta_{u} \leqslant u^{-1 / 2} \quad \text { and } \quad \cos \theta \geqslant \cos \theta_{u} \geqslant \cos \frac{\pi}{4}=\frac{1}{\sqrt{2}} .
$$

and for $\theta \in\left(\theta_{u}, \frac{\pi}{2}\right]$ as $\sin \frac{\pi}{4}=\frac{1}{\sqrt{2}}$ we obtain

$$
\sin \theta \geqslant \min \left\{\frac{2}{\pi} u^{-1 / 2}, \frac{1}{\sqrt{2}}\right\} .
$$

This gives the claim as we may reduce the estimates back to the estimates for $\theta \in\left[0, \frac{\pi}{2}\right]$ by using standard invariance identities for sine and cosine. 
3B. Horizontal angles. When $\theta \in H_{u}$ we will first obtain the following estimate on $\varepsilon$-grids:

Lemma 3.3. Fix $\varepsilon>0$. Almost surely there exists a random constant $C_{\omega}>0$ such that for any $\boldsymbol{k}=$ $u(\cos \theta, \sin \theta) \in \varepsilon \mathbb{Z}^{2} \backslash\{0\}$ with $\theta \in H_{u}$ we have

$$
|\hat{\mu}(\boldsymbol{k})| \leqslant C_{\omega}|\boldsymbol{k}|^{-1 / 2} .
$$

Given $\xi=u(\cos \theta, \sin \theta) \in \mathbb{R}^{2} \backslash\{0\}$ and a realisation $\left(W_{t}\right)$, define a random variable $T=T_{\omega}(\xi) \in[0,1]$, to be the minimum value of $t \in[0,1]$ such that

$$
X_{t}= \begin{cases}-2 \pi\left\lceil u\left(\cos \theta+W_{1} \sin \theta\right)\right\rceil & \text { if } X_{1} \geqslant 0, \\ -2 \pi\left\lfloor u\left(\cos \theta+W_{1} \sin \theta\right)\right\rfloor & \text { if } X_{1}<0 .\end{cases}
$$

Such a time $T$ exists almost surely since $X_{0}=0$ and $X_{t}$ is almost surely continuous (since $W_{t}$ is almost surely continuous). Splitting the integral of $Z_{t}$ up into "complete rotations" and "what is left over", one obtains

$$
\int_{0}^{1} Z_{t} d t=\int_{0}^{T} Z_{t} d t+\int_{T}^{1} Z_{t} d t
$$

For the integral over $[T, 1]$ we get the following estimate.

Lemma 3.4. Almost surely there exists a random constant $C_{\omega}>0$ such that for any $\xi=u(\cos \theta, \sin \theta) \in$ $\mathbb{R}^{2} \backslash\{0\}$ with $\theta \in H_{u}$ we have

$$
\left|\int_{T}^{1} Z_{t} d t\right| \leqslant C_{\omega}|\xi|^{-1 / 2}
$$

Proof. Since $W_{t}$ is almost surely continuous, there almost surely exists a random constant $M_{\omega}>1$ such that $W_{t} \in\left[-M_{\omega}, M_{\omega}\right]$ for all $t \in[0,1]$. Define the real-valued process

$$
Y_{t}:=u\left(t \cos \theta+W_{t} \sin \theta\right),
$$

so $X_{t}=-2 \pi Y_{t}$. Suppose $X_{1} \geqslant 0$. In this case $Y_{T}=\left\lceil Y_{1}\right\rceil \leqslant 0$ and so $Y_{1}+1 \geqslant Y_{T} \geqslant Y_{1}$. Moreover, when $X_{1}<0$ we have $Y_{T}=\left\lfloor Y_{1}\right\rfloor>0$ and $Y_{1} \geqslant Y_{T} \geqslant Y_{1}-1$. Thus no matter what the sign of $X_{1}$ is, we always have almost surely

$$
u\left(\cos \theta+W_{1} \sin \theta\right)+1 \geqslant u\left(T \cos \theta+W_{T} \sin \theta\right) \geqslant u\left(\cos \theta+W_{1} \sin \theta\right)-1 .
$$

Therefore, in the case $\cos \theta>0$ we obtain

$$
T \geqslant 1+W_{1} \frac{\sin \theta}{\cos \theta}-W_{T} \frac{\sin \theta}{\cos \theta}-\frac{1}{u \cos \theta},
$$

and when $\cos \theta<0$ we have

$$
T \geqslant 1+W_{1} \frac{\sin \theta}{\cos \theta}-W_{T} \frac{\sin \theta}{\cos \theta}+\frac{1}{u \cos \theta} .
$$

Since $u \in H_{u}$, Lemma 3.2 together with $W_{t} \in\left[-M_{\omega}, M_{\omega}\right]$ yields

$$
T \geqslant 1-2 \sqrt{2} M_{\omega} u^{-1 / 2}-\frac{\sqrt{2}}{u} \text {. }
$$


Recalling $M_{\omega}>1$ this gives

as required.

$$
\left|\int_{T}^{1} Z_{t} d t\right| \leqslant \int_{T}^{1}\left|Z_{t}\right| d t=1-T \leqslant 4 M_{\omega} u^{-1 / 2}
$$

We now estimate the integral over $[0, T]$, which is where Itô calculus comes into play.

Lemma 3.5. Fix $\varepsilon>0$. Almost surely there exists a random constant $C_{\omega}>0$ such that for any $\boldsymbol{k}=$ $u(\cos \theta, \sin \theta) \in \varepsilon \mathbb{Z}^{2} \backslash\{0\}$ with $\theta \in H_{u}$ we have

$$
\left|\int_{0}^{T} Z_{t} d t\right| \leqslant C_{\omega}|\boldsymbol{k}|^{-1 / 2}
$$

To prove Lemma 3.5, we first need to compute the higher-order moments of the random variable $\int_{0}^{T} Z_{t} d t$.

Lemma 3.6. For any $p \in \mathbb{N}$ and $\xi=u(\cos \theta, \sin \theta) \in \mathbb{R}^{2} \backslash\{0\}$ with $\theta \in H_{u}$, the $(2 p)$-th moment satisfies

$$
\mathbb{E}\left|\int_{0}^{T} Z_{t} d t\right|^{2 p} \leqslant 13 p^{1 / 2} 4^{p}|\xi|^{-p}
$$

Proof. Recall that

$$
X_{t}=-2 \pi u\left(t \cos \theta+W_{t} \sin \theta\right)
$$

is an Itô drift-diffusion process satisfying the stochastic differential equation

$$
d X_{t}=b d t+\sigma d W_{t}
$$

for deterministic and time-independent coefficients $b=-2 \pi u \cos \theta$ and $\sigma=-2 \pi u \sin \theta$. Writing

$$
f(x):=\exp (i x), \quad x \in \mathbb{R},
$$

we have $Z_{t}=f\left(X_{t}\right), f^{\prime}(x)=i \exp (i x)$ and $f^{\prime \prime}(x)=-\exp (i x)$. Thus by complex Itô's lemma (see Lemma 2.3) we have $\mathbb{P}$ almost surely

$$
f\left(X_{T}\right)-f\left(X_{0}\right)=\left(b i-\sigma^{2} / 2\right) \int_{0}^{T} f\left(X_{t}\right) d t+\sigma i \int_{0}^{T} f\left(X_{t}\right) d W_{t} .
$$

Note that $T_{\omega} \leqslant 1$ is random and only $\mathcal{F}_{1}$ measurable; thus it is not a stopping time. However, as Lemma 2.3 is given pathwise, that is, $\mathbb{P}$ almost surely Itô's lemma holds for any time $T \geqslant 0$, then as $T_{\omega}$ is $\mathbb{P}$ almost surely well-defined, we have (3-2) almost surely. Since $X_{0}$ and $X_{T}$ are $2 \pi$ multiples of integers by definition, we have $f\left(X_{T}\right)=f\left(X_{0}\right)=1$. Thus (3-2) gives

$$
\int_{0}^{T} f\left(X_{t}\right) d t=-\frac{\sigma i}{b i-\sigma^{2} / 2} \int_{0}^{T} f\left(X_{t}\right) d W_{t}
$$

Since $b$ and $\sigma$ are deterministic, this yields that the $(2 p)$-th moment satisfies

$$
\mathbb{E}\left|\int_{0}^{T} f\left(X_{t}\right) d t\right|^{2 p}=\left|\frac{\sigma i}{b i-\sigma^{2} / 2}\right|^{2 p} \mathbb{E}\left|\int_{0}^{T} f\left(X_{t}\right) d W_{t}\right|^{2 p} .
$$


Applying the Burkholder-Davis-Gundy inequality (see Lemma 2.4) for the process $\cos X_{t}$ gives

$$
\mathbb{E}\left[\left|\int_{0}^{T} \cos X_{t} d W_{t}\right|^{2 p}\right] \leqslant \mathbb{E}\left[\left(\sup _{0 \leqslant s \leqslant 1}\left|\int_{0}^{s} \cos X_{t} d W_{t}\right|\right)^{2 p}\right] \leqslant 2 \sqrt{10 p} \mathbb{E}\left(\int_{0}^{1} \cos ^{2} X_{t} d t\right)^{p} \leqslant 2 \sqrt{10 p}
$$

since $\cos ^{2} \leqslant 1$. Similar application for the process $\sin X_{t}$ gives

$$
\mathbb{E}\left[\left|\int_{0}^{T} \sin X_{t} d W_{t}\right|^{2 p}\right] \leqslant 2 \sqrt{10 p} .
$$

By Euler's formula, we can write $f\left(X_{t}\right)=\cos X_{t}+i \sin X_{t}$ and so

$$
\int_{0}^{T} f\left(X_{t}\right) d W_{t}=\int_{0}^{T} \cos X_{t} d W_{t}+i \int_{0}^{T} \sin X_{t} d W_{t} .
$$

Hence

$$
\begin{aligned}
\mathbb{E}\left|\int_{0}^{T} f\left(X_{t}\right) d W_{t}\right|^{2 p} & =\mathbb{E}\left[\left(\left|\int_{0}^{T} \cos X_{t} d W_{t}\right|^{2}+\left|\int_{0}^{T} \sin X_{t} d W_{t}\right|^{2}\right)^{p}\right] \\
& \leqslant \mathbb{E}\left[2^{p}\left|\int_{0}^{T} \cos X_{t} d W_{t}\right|^{2 p}+2^{p}\left|\int_{0}^{T} \sin X_{t} d W_{t}\right|^{2 p}\right] \\
& =2^{p}\left(\mathbb{E}\left[\left|\int_{0}^{T} \cos X_{t} d W_{t}\right|^{2 p}\right]+\mathbb{E}\left[\left|\int_{0}^{T} \sin X_{t} d W_{t}\right|^{2 p}\right]\right) \leqslant 2^{p} 4 \sqrt{10 p} .
\end{aligned}
$$

Moreover, as $\theta \in H_{u}$ we have by Lemma 3.2 that $\cos ^{2} \theta \geqslant \frac{1}{2}$ and $\sin ^{2} \theta \leqslant u^{-1}$. Hence

$$
\left|\frac{\sigma i}{b i-\sigma^{2} / 2}\right|^{2}=\frac{\sigma^{2}}{b^{2}+\sigma^{4} / 4} \leqslant \frac{\sigma^{2}}{b^{2}}=\frac{4 \pi^{2} u^{2} \sin ^{2} \theta}{4 \pi^{2} u^{2} \cos ^{2} \theta}=\frac{\sin ^{2} \theta}{\cos ^{2} \theta} \leqslant 2 u^{-1} .
$$

Therefore,

$$
\mathbb{E}\left|\int_{0}^{T} f\left(X_{t}\right) d t\right|^{2 p} \leqslant 4 \sqrt{10 p} 4^{p} \theta^{2 p} \leqslant 13 p^{1 / 2} 4^{p} u^{-p}
$$

as required.

Proof of Lemma 3.5. Fix $\varepsilon>0$. Then for all $k \in \varepsilon \mathbb{Z}^{2} \backslash\{0\}$ define the random variable

$$
I(\boldsymbol{k}):=\left(\int_{0}^{T} Z_{t} d t\right) \cdot \chi_{A}(\boldsymbol{k}),
$$

where $\chi_{A}$ is the indicator function on the set

$$
A:=\left\{\xi=u(\cos \theta, \sin \theta) \in \mathbb{R}^{2} \backslash\{0\}: \theta \in H_{u}\right\} .
$$

Note that $I(\boldsymbol{k})$ is well-defined and finite since $\left|\int_{0}^{T} Z_{t} d t\right| \leqslant 1$ by $|\exp (i x)|=1$. Lemma 3.6 now yields for any $\boldsymbol{k} \in \varepsilon \mathbb{Z}^{2} \backslash\{0\}$ and $p \in \mathbb{N}$ that

$$
\mathbb{E}|I(\boldsymbol{k})|^{2 p} \leqslant 13 p^{1 / 2} 4^{p}|\boldsymbol{k}|^{-p}
$$


as when $\boldsymbol{k} \notin A$ we have $I(\boldsymbol{k}) \equiv 0$. Write $p_{\boldsymbol{k}}=\lfloor\log |\boldsymbol{k}|\rfloor$. Then

$$
\mathbb{E} \sum_{\boldsymbol{k} \in \varepsilon \mathbb{Z}^{2} \backslash\{0\}}|\boldsymbol{k}|^{-3} \frac{|I(\boldsymbol{k})|^{2 p_{\boldsymbol{k}}}}{13 p_{\boldsymbol{k}}^{1 / 2} 4 p_{\boldsymbol{k}}|\boldsymbol{k}|^{-p_{\boldsymbol{k}}}} \leqslant \sum_{\boldsymbol{k} \in \varepsilon \mathbb{Z}^{2} \backslash\{0\}}|\boldsymbol{k}|^{-3}<\infty .
$$

This means that the summands tend to 0 almost surely as $|\boldsymbol{k}| \rightarrow \infty$ and so we can find a random constant $C_{\omega}>0$ such that for all $\boldsymbol{k} \in \varepsilon \mathbb{Z}^{2} \backslash\{0\}$ we have

$$
|\boldsymbol{k}|^{-3} \frac{|I(\boldsymbol{k})|^{2 p_{k}}}{13 p_{\boldsymbol{k}}^{1 / 2} 4 p_{\boldsymbol{k}}|\boldsymbol{k}|^{-p_{\boldsymbol{k}}}} \leqslant C_{\omega} .
$$

Therefore, by possibly making $C_{\omega}$ bigger we obtain

$$
|I(\boldsymbol{k})| \leqslant C_{\omega}|\boldsymbol{k}|^{-1 / 2} \text {. }
$$

This holds for each $\boldsymbol{k} \in \varepsilon \mathbb{Z}^{2} \backslash\{0\}$, so by the definition of $I(\boldsymbol{k})$ we have whenever $\boldsymbol{k}=u(\cos \theta, \sin \theta) \in$ $\varepsilon \mathbb{Z}^{2} \backslash\{0\}$ with $\theta \in H_{u}$ that

as claimed.

$$
\left|\int_{0}^{T} Z_{t} d t\right| \leqslant C_{\omega} u^{-1 / 2}
$$

We are now in position to complete the proof of Lemma 3.3.

Proof of Lemma 3.3. Fix $\varepsilon>0$. By the splitting

$$
\hat{\mu}(\xi)=\int_{0}^{1} Z_{t} d t=\int_{0}^{T} Z_{t} d t+\int_{T}^{1} Z_{t} d t
$$

and Lemmas 3.4 and 3.5, we have that almost surely there exists a constant $C_{\omega}>0$ such that for all $\boldsymbol{k}=u(\cos \theta, \sin \theta) \in \varepsilon \mathbb{Z}^{2} \backslash\{0\}$ with $\theta \in H_{u}$ we have

as required.

$$
|\hat{\mu}(\boldsymbol{k})| \leqslant\left|\int_{0}^{T} Z_{t} d t\right|+\left|\int_{T}^{1} Z_{t} d t\right| \leqslant C_{\omega}|\boldsymbol{k}|^{-1 / 2}
$$

3C. Vertical angles. In this section we apply Kahane's work to obtain Fourier decay estimates when $\theta \in V_{u}$.

Lemma 3.7. Fix $\varepsilon>0$. Almost surely there exists a random constant $C_{\omega}>0$ such that for any $\boldsymbol{k}=$ $u(\cos \theta, \sin \theta) \in \varepsilon \mathbb{Z}^{2} \backslash\{0\}$ with $\theta \in V_{u}$ we have

$$
|\hat{\mu}(\boldsymbol{k})| \leqslant C_{\omega}|\boldsymbol{k}|^{-1 / 2} \sqrt{\log |\boldsymbol{k}|} .
$$

Let us discuss a few estimates obtained in [Kahane 1985b]. Let $v$ be the push-forward of Lebesgue measure on $[0,1]$ under the map $t \mapsto W_{t}$; that is, $v$ is the Brownian image of Lebesgue measure. Kahane established the following:

Theorem 3.8 [Kahane 1985b, page 255]. Almost surely

$$
|\hat{v}(v)| \leqslant O\left(|v|^{-1} \sqrt{\log |v|}\right) \quad \text { as }|v| \rightarrow \infty .
$$


The key ingredient for the proof of Theorem 3.8 was based on establishing the following bound for the higher moments:

Lemma 3.9 [Kahane 1985b, page 254, estimate (2)]. There exists a constant $C>0$ such that for any $v \in \mathbb{R} \backslash\{0\}$ and any $p \in \mathbb{N}$ we have

$$
\mathbb{E}|\hat{v}(v)|^{2 p} \leqslant C^{p} p^{p}|v|^{-2 p}
$$

We can use Lemma 3.9 to give a bound on the higher moments in our setting, but with the price that the exponent will increase from $-2 p$ to $-p$.

Lemma 3.10. There exists a constant $C>0$ such that for any $p \in \mathbb{N}$ and $\xi=u(\cos \theta, \sin \theta) \in \mathbb{R}^{2} \backslash\{0\}$ with $\theta \in V_{u}$ the $(2 p)$-th moment satisfies

$$
\mathbb{E}|\hat{\mu}(\xi)|^{2 p} \leqslant C^{p} p^{p}|\xi|^{-p} .
$$

Proof. Write $\boldsymbol{t}=\left(t_{1}, \ldots, t_{p}\right) \in[0,1]^{p}$ and $d \boldsymbol{t}$ as the Lebesgue measure on $[0,1]^{p}$. Given $\boldsymbol{t}, \boldsymbol{s} \in[0,1]^{p}$, we define

$$
\varphi(\boldsymbol{t}, \boldsymbol{s}):=\sum_{k=1}^{p}\left(t_{k}-s_{k}\right), \quad \psi(\boldsymbol{t}, \boldsymbol{s}):=\sum_{k=1}^{p}\left(W_{t_{k}}-W_{s_{k}}\right), \quad \text { and } \quad \Psi(\boldsymbol{t}, \boldsymbol{s}):=\mathbb{E}|\varphi(\boldsymbol{t}, \boldsymbol{s})|^{2} .
$$

By the definition of $\mu_{\theta}, \mu$ and the Fourier transform, and using the fact that the multivariate process

$$
X(\boldsymbol{t}, \boldsymbol{s}):=-2 \pi \cos (\theta) \varphi(\boldsymbol{t}, \boldsymbol{s})-2 \pi \sin (\theta) \psi(\boldsymbol{t}, \boldsymbol{s})
$$

is Gaussian with mean $-2 \pi \cos (\theta) \varphi(\boldsymbol{t}, \boldsymbol{s})$ and variance $4 \pi^{2} \sin ^{2}(\theta) \Psi(\boldsymbol{t}, \boldsymbol{s})$, we have through Fubini's theorem and the formula for the characteristic function that

$$
\begin{aligned}
\mathbb{E}|\hat{\mu}(\xi)|^{2 p} & =\mathbb{E} \int_{[0,1]^{p}} \int_{[0,1]^{p}} \exp (-2 \pi i u(\cos (\theta) \varphi(\boldsymbol{t}, \boldsymbol{s})+\sin (\theta) \psi(\boldsymbol{t}, \boldsymbol{s}))) d \boldsymbol{t} d \boldsymbol{s} \\
& =\int_{[0,1]^{p}} \int_{[0,1]^{p}} \mathbb{E} \exp (i u X(\boldsymbol{t}, \boldsymbol{s})) d \boldsymbol{t} d \boldsymbol{s} \\
& =\int_{[0,1]^{p}} \int_{[0,1]^{p}} \exp \left(-2 \pi i \cos (\theta) u \varphi(\boldsymbol{t}, \boldsymbol{s})-2 \pi^{2}|u \sin (\theta)|^{2} \Psi(\boldsymbol{t}, \boldsymbol{s})\right) d \boldsymbol{t} d \boldsymbol{s} .
\end{aligned}
$$

Thus by taking absolute values inside the integrals, and observing that $|\exp (i x)|=1$ for any $x \in \mathbb{R}$, we obtain

$$
\mathbb{E}|\hat{\mu}(\xi)|^{2 p} \leqslant \int_{[0,1]^{p}} \int_{[0,1]^{p}} \exp \left(-2 \pi^{2}|u \sin (\theta)|^{2} \Psi(\boldsymbol{t}, \boldsymbol{s})\right) d \boldsymbol{t} d \boldsymbol{s} .
$$

On the other hand, by doing the expansion again for the Fourier transform $\hat{v}$ of the image measure $v$ at $v:=u \sin (\theta) \in \mathbb{R} \backslash\{0\}$ we see that

$$
\mathbb{E}|\hat{v}(v)|^{2 p}=\mathbb{E} \int_{[0,1]^{p}} \int_{[0,1]^{p}} \exp (-2 \pi i v \psi(\boldsymbol{t}, \boldsymbol{s})) d \boldsymbol{t} d \boldsymbol{s}=\int_{[0,1]^{p}} \int_{[0,1]^{p}} \exp \left(-2 \pi^{2} v^{2} \Psi(\boldsymbol{t}, \boldsymbol{s})\right) d \boldsymbol{t} d \boldsymbol{s},
$$

which is equal to (3-3). Thus by Lemma 3.9 we have

$$
\mathbb{E}|\hat{\mu}(\xi)|^{2 p} \leqslant C^{p} p^{p}|v|^{-2 p} .
$$


Since $\theta \in V_{u}$ we have $|\sin \theta| \geqslant \min \left\{\frac{2}{\pi} u^{-1 / 2}, \frac{1}{\sqrt{2}}\right\}$. When $|\sin \theta| \geqslant \frac{1}{\sqrt{2}}$ we obtain

$$
C^{p} p^{p}|v|^{-2 p} \leqslant(2 C)^{p} p^{p} u^{-2 p} \leqslant(2 C)^{p} p^{p} u^{-p} .
$$

On the other hand, if $|\sin \theta| \geqslant \frac{2}{\pi} u^{-1 / 2}$ we have

$$
C^{p} p^{p}|v|^{-2 p} \leqslant C^{p} p^{p}\left(2 u^{-1 / 2} / \pi\right)^{-2 p} u^{-2 p} \leqslant\left(C \pi^{2} / 4\right)^{p} p^{p} u^{-p} .
$$

Now we can complete the proof of Lemma 3.7 for vertical directions:

Proof of Lemma 3.7. Fix $\varepsilon>0$. Then for all $\boldsymbol{k}=u(\cos \theta, \sin \theta) \in \varepsilon \mathbb{Z}^{2} \backslash\{0\}$ define the random variable

$$
F(\boldsymbol{k}):=\hat{\mu}(\boldsymbol{k}) \chi_{B}(\boldsymbol{k}),
$$

where

$$
B:=\left\{\xi=u(\cos \theta, \sin \theta) \in \mathbb{R}^{2} \backslash\{0\}: \theta \in V_{u}\right\} .
$$

Now $F(\boldsymbol{k})$ is a well-defined finite random variable as $|\hat{\mu}(\boldsymbol{k})| \leqslant 1$ for any $\boldsymbol{k}$. From Lemma 3.10 we obtain for any $\boldsymbol{k} \in \varepsilon \mathbb{Z}^{2} \backslash\{0\}$ and $p \in \mathbb{N}$ that

$$
\mathbb{E}|F(\boldsymbol{k})|^{2 p} \leqslant C^{p} p^{p}|\boldsymbol{k}|^{-p}
$$

Write $p_{k}=\lfloor\log |\boldsymbol{k}|\rfloor$. Then

$$
\mathbb{E} \sum_{\boldsymbol{k} \in \varepsilon \mathbb{Z}^{2} \backslash\{0\}}|\boldsymbol{k}|^{-3} \frac{|F(\boldsymbol{k})|^{2 p_{\boldsymbol{k}}}}{C^{p_{\boldsymbol{k}}} p_{\boldsymbol{k}}^{p_{\boldsymbol{k}}}|\boldsymbol{k}|^{-p_{\boldsymbol{k}}}} \leqslant \sum_{\boldsymbol{k} \in \varepsilon \mathbb{Z}^{2} \backslash\{0\}}|\boldsymbol{k}|^{-3}<\infty .
$$

This means that the summands tend to 0 almost surely as $|\boldsymbol{k}| \rightarrow \infty$ and so we can find a random constant $C_{\omega}>0$ such that for all $\boldsymbol{k} \in \varepsilon \mathbb{Z}^{2} \backslash\{0\}$ we have

$$
|\boldsymbol{k}|^{-3} \frac{|F(\boldsymbol{k})|^{2 p_{k}}}{C^{p_{k}} p_{\boldsymbol{k}}^{p_{\boldsymbol{k}}}|\boldsymbol{k}|^{-p_{k}}} \leqslant C_{\omega} .
$$

Thus possibly making $C_{\omega}$ bigger, this yields

$$
|F(\boldsymbol{k})| \leqslant C_{\omega}|\boldsymbol{k}|^{-1 / 2} \sqrt{\log |\boldsymbol{k}|} .
$$

Now this holds for each $\boldsymbol{k} \in \varepsilon \mathbb{Z}^{2} \backslash\{0\}$, so by the definition of $F(\boldsymbol{k})$ we have, whenever $\boldsymbol{k}=u(\cos \theta, \sin \theta) \in$ $\varepsilon \mathbb{Z}^{2} \backslash\{0\}$ with $\theta \in V_{u}$, that

$$
|\hat{\mu}(\boldsymbol{k})| \leqslant C_{\omega}|\boldsymbol{k}|^{-1 / 2} \sqrt{\log |\boldsymbol{k}|}
$$

as claimed.

3D. From lattices to $\mathbb{R}^{2}$. We can now complete the proof of the main theorem. For this purpose, we need the following comparison lemma used by Kahane that allows us to pass from convergence on lattices for Fourier transforms to the whole space:

Lemma 3.11 [Kahane 1985b, Lemma 1, page 252]. Suppose $\tau$ is a measure on $\mathbb{R}^{2}$ with support in $(-1,1)^{2}$. Suppose $\varphi, \psi:(0, \infty) \rightarrow(0, \infty)$ are decreasing as $t \rightarrow \infty$ with the doubling properties

$$
\varphi(t / 2)=O(\varphi(t)) \quad \text { and } \quad \psi(t / 2)=O(\psi(t)) \quad \text { as } t \rightarrow \infty .
$$


If the Fourier transform of $\tau$ along the integer lattice $\mathbb{Z}^{2}$ satisfies

then

$$
|\hat{\tau}(\boldsymbol{n})|=O\left(\frac{\varphi(|\boldsymbol{n}|)}{\psi(|\boldsymbol{n}|)}\right) \quad \text { as }|\boldsymbol{n}| \rightarrow \infty,
$$

$$
|\hat{\tau}(\xi)|=O\left(\frac{\varphi(|\xi|)}{\psi(|\xi|)}\right) \quad \text { as }|\xi| \rightarrow \infty .
$$

Proof of Theorem 1.3. Combining Lemmas 3.7 and 3.3 we have that for any $\varepsilon>0$, almost surely, there exists some random constant $C_{\omega}>0$ such that for any $\boldsymbol{k}=u(\cos \theta, \sin \theta) \in \varepsilon \mathbb{Z}^{2} \backslash\{0\}$ we have

$$
|\hat{\mu}(\boldsymbol{k})| \leqslant C_{\omega}|\boldsymbol{k}|^{-1 / 2} \sqrt{\log |\boldsymbol{k}|} .
$$

Define a measure $\tau_{\varepsilon}$ on $\mathbb{R}^{2}$ such that

$$
\hat{\tau}_{\varepsilon}(\xi):=\hat{\mu}(\varepsilon \xi), \quad \xi \in \mathbb{R}^{2} .
$$

By the almost sure continuity of $W_{t}$, we have that there exists a random constant $M_{\omega}>0$ such that the diameter of the support of $\mu$ is at most $M_{\omega}$ almost surely. Taking an intersection of the events that (3-4) holds for $\varepsilon=1 / n$ over all $n \in \mathbb{N}$ allows us to find a random $\varepsilon=\varepsilon_{\omega}>0$ such that $\mu$ is supported on a set of diameter strictly less than $1 / \varepsilon$ and (3-4) holds almost surely with this $\varepsilon$. This guarantees that the measure $\tau_{\varepsilon}$ is supported on $(-1,1)^{2}$ and so applying Lemma 3.11 with the measure $\tau=\tau_{\varepsilon}$ and the maps $\varphi(t):=\sqrt{\log t}$ and $\psi(t):=t^{1 / 2}$ gives the claim.

\section{Acknowledgements}

We thank Tuomas Orponen for useful discussions during the preparation of this manuscript. We are also grateful to an anonymous referee for comments and suggestions which improved the focus of the paper. Finally, we thank The Hebrew University of Jerusalem and The University of Manchester for hosting us for research visits during the writing of this paper.

\section{References}

[Adler 1977] R. J. Adler, "Hausdorff dimension and Gaussian fields", Ann. Probability 5:1 (1977), 145-151. MR Zbl

[Beffara 2008] V. Beffara, "The dimension of the SLE curves”, Ann. Probab. 36:4 (2008), 1421-1452. MR Zbl

[Bluhm 1998] C. Bluhm, "On a theorem of Kaufman: Cantor-type construction of linear fractal Salem sets", Ark. Mat. 36:2 (1998), 307-316. MR Zbl

[Burkholder et al. 1972] D. L. Burkholder, B. J. Davis, and R. F. Gundy, "Integral inequalities for convex functions of operators on martingales", pp. 223-240 in Proceedings of the Sixth Berkeley Symposium on Mathematical Statistics and Probability, II: Probability theory (Berkeley, CA, 1970/1971), Univ. California Press, Berkeley, CA, 1972. MR Zbl

[Chan et al. 2016] V. Chan, I. Łaba, and M. Pramanik, "Finite configurations in sparse sets", J. Anal. Math. 128 (2016), 289-335. MR Zbl

[Davenport et al. 1963] H. Davenport, P. Erdős, and W. J. LeVeque, "On Weyl's criterion for uniform distribution", Michigan Math. J. 10 (1963), 311-314. MR Zbl

[Ekström et al. 2015] F. Ekström, T. Persson, and J. Schmeling, "On the Fourier dimension and a modification", J. Fractal Geom. 2:3 (2015), 309-337. MR Zbl 
[Fouché and Mukeru 2013] W. L. Fouché and S. Mukeru, "On the Fourier structure of the zero set of fractional Brownian motion", Statist. Probab. Lett. 83:2 (2013), 459-466. MR Zbl

[Fraser et al. 2014] J. M. Fraser, T. Orponen, and T. Sahlsten, "On Fourier analytic properties of graphs", Int. Math. Res. Not. 2014:10 (2014), 2730-2745. MR Zbl

[Jordan and Sahlsten 2016] T. Jordan and T. Sahlsten, "Fourier transforms of Gibbs measures for the Gauss map", Math. Ann. 364:3-4 (2016), 983-1023. MR Zbl

[Kahane 1966a] J.-P. Kahane, "Images browniennes des ensembles parfaits", C. R. Acad. Sci. Paris Sér. A-B 263 (1966), A613-A615. MR Zbl

[Kahane 1966b] J.-P. Kahane, "Images d'ensembles parfaits par des séries de Fourier gaussiennes”, C. R. Acad. Sci. Paris Sér. $A-B 263$ (1966), A678-A681. MR Zbl

[Kahane 1985a] J.-P. Kahane, "Ensembles aléatoires et dimensions", pp. 65-121 in Recent progress in Fourier analysis (El Escorial, 1983), edited by I. Peral and J. L. Rubio de Francia, North-Holland Math. Stud. 111, North-Holland, Amsterdam, 1985. MR Zbl

[Kahane 1985b] J.-P. Kahane, Some random series of functions, 2nd ed., Cambridge Studies in Advanced Mathematics 5, Cambridge University Press, 1985. MR Zbl

[Kahane 1993] J.-P. Kahane, "Fractals and random measures", Bull. Sci. Math. 117:1 (1993), 153-159. MR Zbl

[Karatzas and Shreve 1991] I. Karatzas and S. E. Shreve, Brownian motion and stochastic calculus, 2nd ed., Graduate Texts in Mathematics 113, Springer, 1991. MR Zbl

[Kaufman 1980] R. Kaufman, "Continued fractions and Fourier transforms”, Mathematika 27:2 (1980), 262-267. MR Zbl

[Kaufman 1981] R. Kaufman, "On the theorem of Jarník and Besicovitch”, Acta Arith. 39:3 (1981), 265-267. MR Zbl

[Körner 2011] T. W. Körner, "Hausdorff and Fourier dimension”, Studia Math. 206:1 (2011), 37-50. MR Zbl

[Łaba and Pramanik 2009] I. Łaba and M. Pramanik, "Arithmetic progressions in sets of fractional dimension", Geom. Funct. Anal. 19:2 (2009), 429-456. MR Zbl

[Mattila 1995] P. Mattila, Geometry of sets and measures in Euclidean spaces: fractals and rectifiability, Cambridge Studies in Advanced Mathematics 44, Cambridge University Press, 1995. MR Zbl

[Mattila 2015] P. Mattila, Fourier analysis and Hausdorff dimension, Cambridge Studies in Advanced Mathematics 150, Cambridge University Press, 2015. MR Zbl

[McKean 1955] H. P. McKean, Jr., "Hausdorff-Besicovitch dimension of Brownian motion paths", Duke Math. J. 22 (1955), 229-234. MR Zbl

[Peres and Sousi 2016] Y. Peres and P. Sousi, "Dimension of fractional Brownian motion with variable drift", Probab. Theory Related Fields 165:3-4 (2016), 771-794. MR Zbl

[Perkins 1981] E. Perkins, "The exact Hausdorff measure of the level sets of Brownian motion”, Z. Wahrsch. Verw. Gebiete 58:3 (1981), 373-388. MR Zbl

[Peškir 1996] G. Peškir, “On the exponential Orlicz norms of stopped Brownian motion”, Studia Math. 117:3 (1996), $253-273$. MR Zbl

[Queffélec and Ramaré 2003] M. Queffélec and O. Ramaré, "Analyse de Fourier des fractions continues à quotients restreints", Enseign. Math. (2) 49:3-4 (2003), 335-356. MR Zbl

[Salem 1951] R. Salem, "On singular monotonic functions whose spectrum has a given Hausdorff dimension", Ark. Mat. 1 (1951), 353-365. MR Zbl

[Shieh and Xiao 2006] N.-R. Shieh and Y. Xiao, "Images of Gaussian random fields: Salem sets and interior points", Studia Math. 176:1 (2006), 37-60. MR Zbl

[Shmerkin 2017] P. Shmerkin, "Salem sets with no arithmetic progressions", Int. Math. Res. Not. 2017:7 (2017), 1929-1941. MR

[Taylor 1953] S. J. Taylor, "The Hausdorff $\alpha$-dimensional measure of Brownian paths in $n$-space", Proc. Cambridge Philos. Soc. 49 (1953), 31-39. MR Zbl

[Taylor 1955] S. J. Taylor, "The $\alpha$-dimensional measure of the graph and set of zeros of a Brownian path", Proc. Cambridge Philos. Soc. 51 (1955), 265-274. MR Zbl 
Received 28 Mar 2016. Revised 19 Jul 2017. Accepted 5 Sep 2017.

JONATHAN M. FRASER: jmf32@st-andrews.ac.uk

School of Mathematics and Statistics, University of St Andrews, St Andrews, United Kingdom

TUOMAS SAHLSTEN: tuomas.sahlsten@manchester.ac.uk

School of Mathematics, University of Manchester, Manchester, United Kingdom 


\title{
Analysis \& PDE
}

msp.org/apde

\section{EDITORS}

\author{
EDITOR-IN-CHIEF \\ Patrick Gérard \\ patrick.gerard@math.u-psud.fr \\ Université Paris Sud XI \\ Orsay, France
}

BOARD OF EDITORS

Nicolas Burq Université Paris-Sud 11, France nicolas.burq@math.u-psud.fr

Massimiliano Berti Scuola Intern. Sup. di Studi Avanzati, Italy berti@sissa.it

Sun-Yung Alice Chang Princeton University, USA chang@math.princeton.edu

Michael Christ University of California, Berkeley, USA mchrist@math.berkeley.edu

$\begin{aligned} \text { Charles Fefferman } & \begin{array}{l}\text { Princeton University, USA } \\ \text { cf@ math.princeton.edu }\end{array} \\ \text { Ursula Hamenstaedt } & \begin{array}{l}\text { Universität Bonn, Germany } \\ \text { ursula@ math.uni-bonn.de }\end{array} \\ \text { Vaughan Jones } & \begin{array}{l}\text { U.C. Berkeley \& Vanderbilt University } \\ \text { vaughan.f.jones@ @anderbilt.edu }\end{array} \\ \text { Vadim Kaloshin } & \begin{array}{l}\text { University of Maryland, USA } \\ \text { vadim.kaloshin@gmail.com }\end{array} \\ \text { Herbert Koch } & \text { Universität Bonn, Germany } \\ & \text { koch@math.uni-bonn.de } \\ \text { Izabella Laba } & \begin{array}{l}\text { University of British Columbia, Canada } \\ \text { ilaba@math.ubc.ca }\end{array} \\ \text { Gilles Lebeau } & \begin{array}{l}\text { Université de Nice Sophia Antipolis, France } \\ \text { lebeau@ unice.fr }\end{array}\end{aligned}$

Richard B. Melrose Massachussets Inst. of Tech., USA rbm@math.mit.edu

Frank Merle Université de Cergy-Pontoise, France Frank.Merle@u-cergy.fr

William Minicozzi II Johns Hopkins University, USA minicozz@math.jhu.edu

Clément Mouhot Cambridge University, UK c.mouhot@dpmms.cam.ac.uk
Werner Müller Universität Bonn, Germany mueller@math.uni-bonn.de

Gilles Pisier Texas A\&M University, and Paris 6 pisier@math.tamu.edu

Tristan Rivière ETH, Switzerland riviere@math.ethz.ch

Igor Rodnianski Princeton University, USA irod@math.princeton.edu

Wilhelm Schlag University of Chicago, USA schlag@math.uchicago.edu

Sylvia Serfaty New York University, USA serfaty@cims.nyu.edu

Yum-Tong Siu Harvard University, USA siu@math.harvard.edu

Terence Tao University of California, Los Angeles, USA tao@math.ucla.edu

Michael E. Taylor Univ. of North Carolina, Chapel Hill, USA met@math.unc.edu

Gunther Uhlmann

András Vasy

University of Washington, USA gunther@math.washington.edu

Stanford University, USA andras@math.stanford.edu

Dan Virgil Voiculescu University of California, Berkeley, USA dvv@math.berkeley.edu

Steven Zelditch Northwestern University, USA zelditch@math.northwestern.edu

Maciej Zworski University of California, Berkeley, USA zworski@math.berkeley.edu

PRODUCTION

production@msp.org

Silvio Levy, Scientific Editor

See inside back cover or msp.org/apde for submission instructions.

The subscription price for 2018 is US \$275/year for the electronic version, and \$480/year (+\$55, if shipping outside the US) for print and electronic. Subscriptions, requests for back issues from the last three years and changes of subscriber address should be sent to MSP.

Analysis \& PDE (ISSN 1948-206X electronic, 2157-5045 printed) at Mathematical Sciences Publishers, 798 Evans Hall \#3840, c/o University of California, Berkeley, CA 94720-3840, is published continuously online. Periodical rate postage paid at Berkeley, CA 94704, and additional mailing offices.

APDE peer review and production are managed by EditFlow ${ }^{\circledR}$ from MSP.

\section{PUBLISHED BY}

mathematical sciences publishers

nonprofit scientific publishing

http://msp.org/

(C) 2018 Mathematical Sciences Publishers 


\section{ANALYSIS \& PDE}

\section{Volume $11 \quad$ No. $1 \quad 2018$}

Analytic torsion, dynamical zeta functions, and the Fried conjecture SHU SHEN

Existence theorems of the fractional Yamabe problem

Seunghyeok Kim, Monica Musso and Juncheng WeI

On the Fourier analytic structure of the Brownian graph

JONATHAN M. FrASER and TUOMAS SAHLSTEN

Nodal geometry, heat diffusion and Brownian motion

Bogdan GeORgIEV and MAYukh MukHERJEe

A normal form à la Moser for diffeomorphisms and a generalization of Rüssmann's translated curve theorem to higher dimensions

JESSICA Elis A MASSETTI

Global results for eikonal Hamilton-Jacobi equations on networks

ANTONIO SICONOLFI and ALFONSO SORRENTINO

High-frequency approximation of the interior Dirichlet-to-Neumann map and applications to

the transmission eigenvalues

GEORGI VODEV

Hardy-Littlewood inequalities on compact quantum groups of Kac type

SANG-GYUN YOUN 